\title{
HIPERPLASIA DE RESTOS ADRENALES EN EL TESTÍCULO: UNA CAUSA INFRECUENTE DE INFERTILIDAD MASCULINA
}

\author{
P. SAN MIGUEL FRAILE, G. FERNÁNDEZ FERNÁNDEZ*, F. MEIJIDE RICO**, \\ I. ANTÓN BADIOLA, J.A. ORTIZ-REY, C. ÁLVAREZ ÁLVAREZ,
} A. DE LA FUENTE BUCETA

\begin{abstract}
Servicio de Anatomía Patológica. *Servicio de Radiología. **Servicio de Urología. Centro Médico POVISA. Vigo (Pontevedra).
\end{abstract}

Actas Urol Esp. 27 (3): 234-239, 2003

\section{RESUMEN \\ "HIPERPLASIA DE RESTOS ADRENALES EN EL TESTÍCULO: UNA CAUSA INFRECUENTE DE INFERTILIDAD MASCULINA"}

Presentamos un caso de azoospermia secretora y masas testiculares bilaterales secundarias a hiperplasia adrenal congénita por déficit de 21-hidroxilasa en un varón de 37 años. La biopsia testicular fue informada como tumor de células de Leydig, pero se reconsideró el diagnóstico después de la información clínica, siendo el diagnóstico final de hiperplasia de restos adrenales en el testículo. Comentamos el diagnóstico diferencial con el tumor de células de Leydig insistiendo en la necesidad de diagnosticar esta lesión con una adecuada correlación clínica, analítica, radiológica e histológica.

PALABRAS CLAVE: Infertilidad. Hiperplasia de restos adrenales. Hiperplasia adrenal congénita. Tumor de células de Leydig. Tumores de testículo.

\begin{abstract}
"TESTICULAR ADRENAL REST TISSUE HYPERPLASIA: AN UNUSUAL CAUSE OF MALE INFERTILITY"

We report the case of a 37-year-old man with infertility caused by bilateral testicular masses secondary to congenital adrenal hyperplasia (21-hydroxylase deficiency). Testicular biopsy was done and its was initially interpreted as Leydig cell tumor but after clinical information was histologically reclassified as tumor of the adrenogenital syndrome. The differential diagnosis with Leydig cell tumor is discussed and it must be established through the clinical, biochemical, radiological and pathological features.
\end{abstract}

KEY WORDS: Infertility. Adrenal rest hyperplasia. Congenital adrenal hyperplasia. Leydig cell tumor. Testicular neoplasm.

$\mathrm{L}$ a hiperplasia adrenal congénita por déficit de 21-hidroxilasa es una enfermedad monogénica infrecuente que se hereda de forma autosómica recesiva ${ }^{1}$. Las manifestaciones clínicas en estos pacientes son variables, aunque suelen presentar signos de hiperandrogenismo después del nacimiento, que pueden hacerse evidentes durante la infancia, adolescencia e incluso en la edad adulta dependiendo del grado de afectación, así como del seguimiento clínico del paciente ${ }^{2,3}$.
Una manifestación clínica infrecuente en estos pacientes es el agrandamiento testicular bilateral, que generalmente refleja un mal cumplimiento terapéutico y que requiere un diagnóstico precoz por su posible evolución hacia la infertilidad ${ }^{3-6}$.

Presentamos un caso de hiperplasia de restos adrenales testiculares en un paciente con hiperplasia adrenal congénita por déficit de 21-hidroxilasa sin seguimiento médico, que se manifestó clínicamente como infertilidad y agrandamiento 
testicular bilateral. Comentamos las características clínicas, radiológicas e histológicas así como los problemas diagnósticos que plantea esta enfermedad, sobre todo con respecto al tumor de células de Leydig.

\section{CASO CLÍNICO}

Paciente de 37 años de edad con antecedentes de hiperplasia adrenal congénita por déficit de 21hidroxilasa y en tratamiento supresor con hidrocortisona, cuyo seguimiento terapéutico y clínico no fue óptimo. Actualmente el paciente acude al Servicio de Urología de nuestro Hospital para estudio de infertilidad.

En la exploración clínica destaca obesidad grado III de distribución central y un aumento bilateral de ambos testículos que son de consistencia dura y configuración nodular.

Los datos analíticos quedan reflejados en la Tabla I, destacando unos niveles aumentados de delta-4-androstendiona y 17 hidroxi-progesterona y niveles disminuidos de cortisol.

El eco-doppler testicular demostró testes polilobulados de $10 \mathrm{~cm}$ de eje longitudinal con ecogeneidad heterogénea, con ligera fibrosis y aumento de la vascularización, observándose vasos que penetran en el tejido sin cambiar de curso o calibre. En la resonancia magnética nuclear (RMN) se evidenció testes de configuración nodular con áreas hipodensas en T2 e isodensas en T1 (Fig. 1) y una hiperplasia de ambas suprarrenales (Fig. 2).

\section{TABLA I}

DATOS ANALÍTICOS DEL PACIENTE

\begin{tabular}{|l|l|l|}
\hline Hormona & $\begin{array}{l}\text { Valores } \\
\text { Paciente }\end{array}$ & Valores normales \\
\hline $\begin{array}{l}\text { Delta 4 } \\
\text { androstendiona }\end{array}$ & $\mathbf{1 6 , 5} \mathbf{~ n g r / ~} \mathbf{m l}$ & $0,3-2,9 \mathrm{ngr} / \mathrm{ml}$ \\
\hline Testosterona & $8,31 \mathrm{ngr} / \mathrm{ml}$ & $3-11 \mathrm{ngr} / \mathrm{ml}$ \\
\hline $\mathbf{1 7 - O H}-$ Progesterona & $\mathbf{1 8 8 , 2 6} \mathbf{~ n g r / ~} \mathbf{~ m l}$ & $0,5-2,4 \mathrm{ngr} / \mathrm{ml}$ \\
\hline ACTH & $40,6 \mathrm{ngr} / \mathrm{ml}$ & $\begin{array}{l}\text { Mañana: } 52 \mathrm{ngr} / \mathrm{ml} \\
\text { Tarde: } 20 \mathrm{ngr} / \mathrm{ml}\end{array}$ \\
\hline Cortisol & $\mathbf{6 ~} \mathbf{m c g r} / \mathbf{d l}$ & $\begin{array}{l}\text { Mañana: } 7 \mathrm{mcgr} / \mathrm{dl} \\
\text { Tarde: } 25 \mathrm{mcgr} / \mathrm{dl}\end{array}$ \\
\hline FSH & $30,06 \mathrm{mUI} / \mathrm{ml}$ & $1-8 \mathrm{mUI} / \mathrm{ml}$ \\
\hline
\end{tabular}
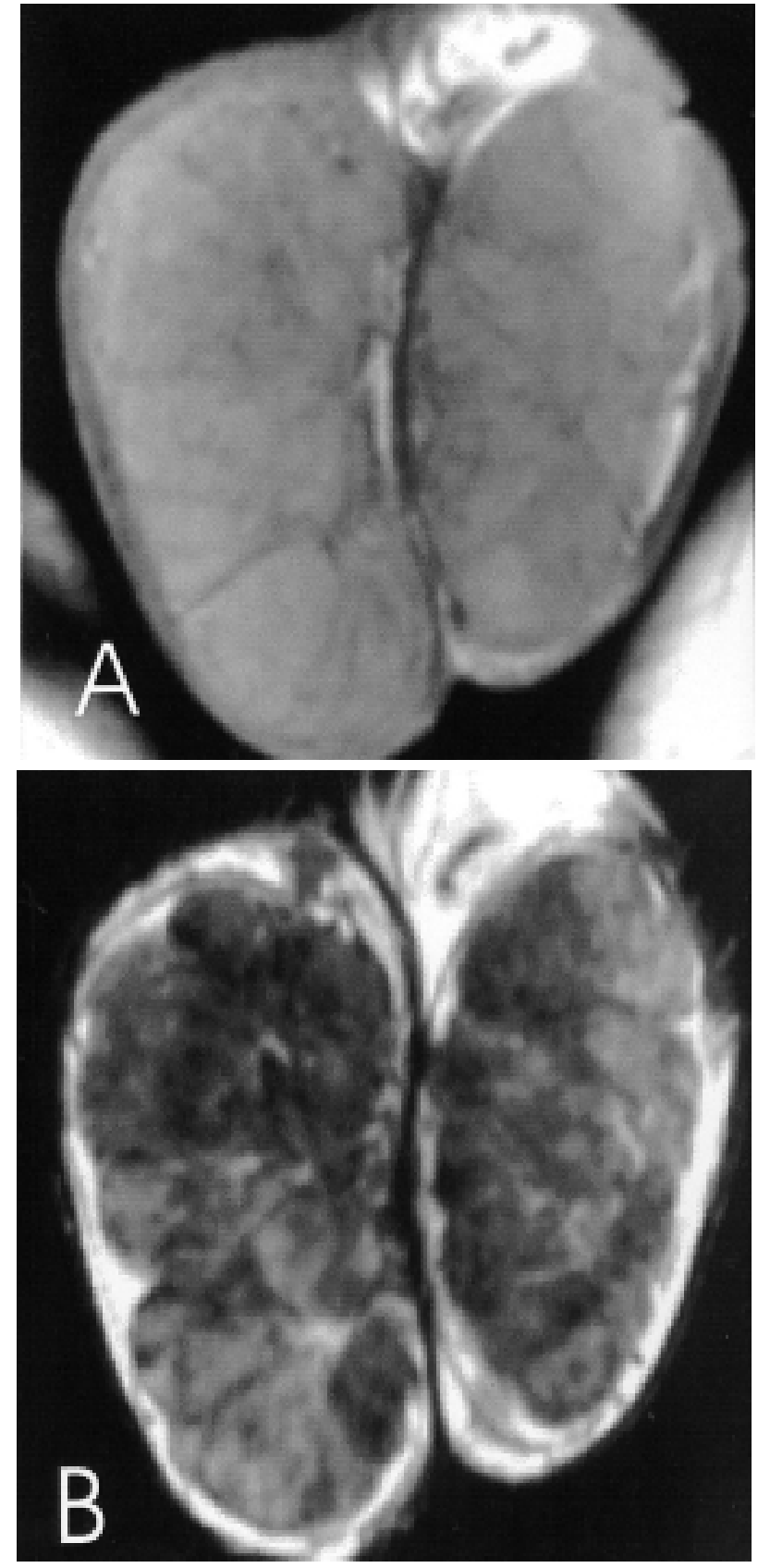

FIGURA 1. Resonancia magnética testicular: (A) Secuencia potenciada T1 apreciándose un aumento de tamaño de ambos testes, con contornos polibulados. (B) Secuencia potenciada en $\mathrm{T} 2$ mostrando una sustitución de parénquima testicular por lesiones nodulares.

Con los datos clínicos y radiológicos se realizó una biopsia del testículo derecho, mediante incisión escrotal, apertura de la túnica vaginal y enucleación de algunos de los nódulos testiculares a través de una incisión en el testículo derecho de aproximadamente $3 \mathrm{~cm}$ de longitud, para búsqueda de espermatozoides en el testículo. 


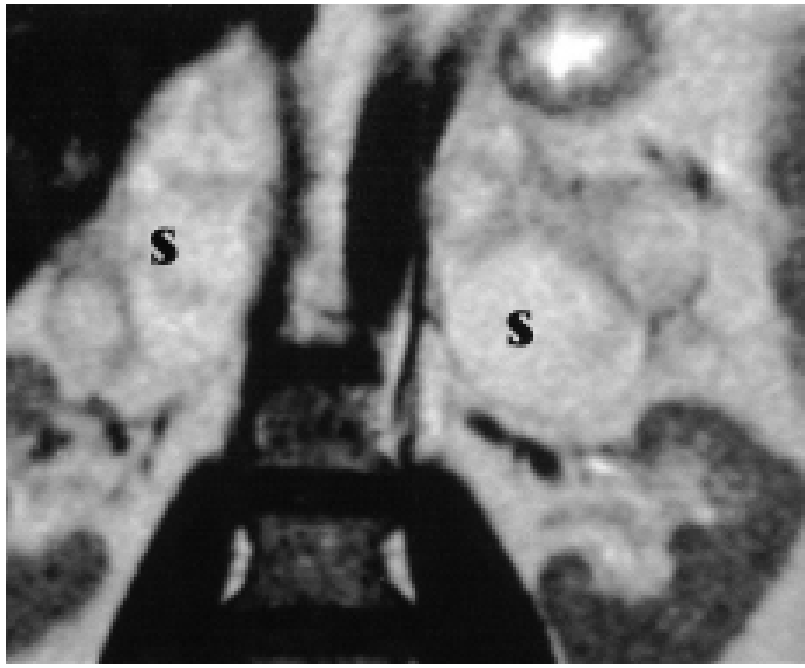

FIGURA 2. Resonancia magnética abdominal. Secuencia potenciada en $\mathrm{T} 1$. Importante aumento de tamaño de ambas glándulas suprarrenales en relación con hiperplasia suprarrenal (S).

En el Servicio de Anatomía Patológica se recibieron varios fragmentos irregulares de coloración blanquecina y configuración nodular, que pesaban en conjunto $26 \mathrm{~g}$ y medía el fragmento de mayor tamaño $3 \mathrm{~cm}$ de diámetro.

Microscópicamente se apreció una formación homogénea de configuración nodular, tabicada por gruesos haces de tejido mesenquimal en donde se observaban frecuentes vasos y un ligero infiltrado inflamatorio predominantemente linfocitario, que adquiría focalmente una configuración nodular. La tumoración estaba constituida por una población monomorfa de células con citoplasma amplio eosinófilo que contenían gran cantidad de pigmento de tipo lipofucsina (Fig. 3). El núcleo mostraba ligera atipia, era de localización central y presentaba un nucleolo patente. El índice mitótico fue muy bajo y no se observaron cristales de Reinke a pesar de realizar una búsqueda exhaustiva. Con las técnicas de inmunohistoquímica se demostró positividad para la inhibina y sinaptofisina, y negatividad para la vimentina y queratina (CAM 5.2).

Con estas características histológicas, y prescindiendo en gran parte de los datos clínicos y analíticos descritos anteriormente, nuestro diagnóstico sería de tumor de células de Leydig, pero los datos clínicos que fueron fundamentales para establecer el diagnóstico definitivo como es el aumento llamativo de los precursores del cortisol en vena espermática, y la reducción del tamaño testicular después de instaurar el tratamiento supresor con hidrocortisona.

Estos dos datos clínicos junto con la presencia de unos rasgos histológicos compatibles con hiperplasia de restos adrenales, e histológicamente por sí solos indistinguibles del tumor de células de Leydig, hicieron que el diagnóstico definitivo de este paciente fuera hiperplasia de restos adrenales en paciente con hiperplasia adrenal congénita por déficit de 21-hidroxilasa.

\section{DISCUSIÓN}

La hiperplasia suprarrenal congénita por déficit de 21-hidroxilasa es la enfermedad hereditaria monogenética autosómica recesiva más frecuen$t^{1}$. El diagnóstico se basa fundamentalmente en criterios analíticos, al producirse un acúmulo de los precursores de cortisol y aldosterona situados por encima del bloqueo enzimático ${ }^{4,6}$ (Tabla II). El déficit de la 21-hidroxilasa, como el de nuestro paciente, se caracteriza por un aumento de los niveles de 17-OH-progesterona acompañado de una disminución de cortisol y un aumento de ACTH (Tabla I).

Los varones con esta enfermedad no tratados o mal controlados presentan muy frecuentemente hiperplasia de restos adrenales en el testículo que en muchas ocasiones pasa desapercibida, ya

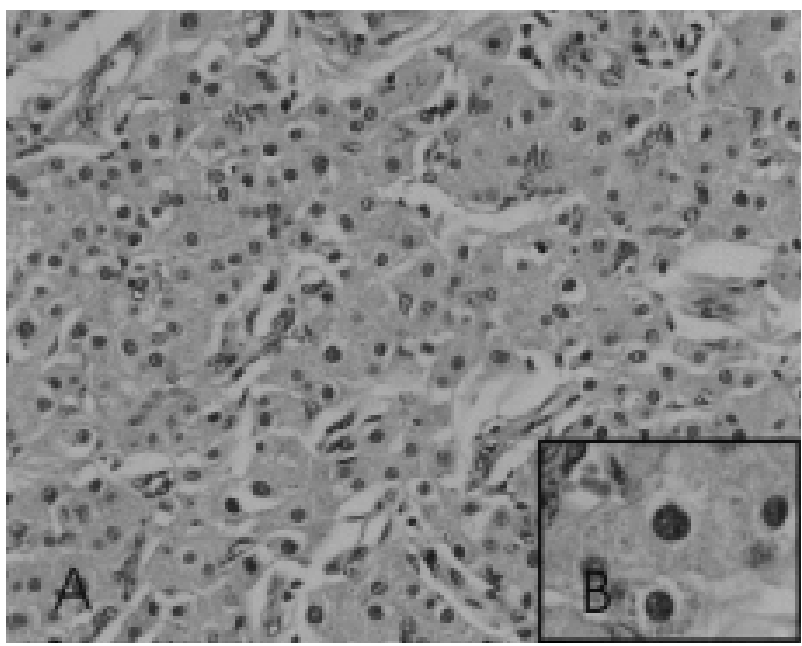

FIGURA 3. A) (x $400 \mathrm{HE}$ ). Biopsia de testiculo constituido por una proliferación homogénea de células con escasa atipia indistinguibles histológicamente del tumor de células de Leydig. B) (x 400 H\&E). Presencia de gran cantidad de lipofucsina en el citoplasma de las células. 


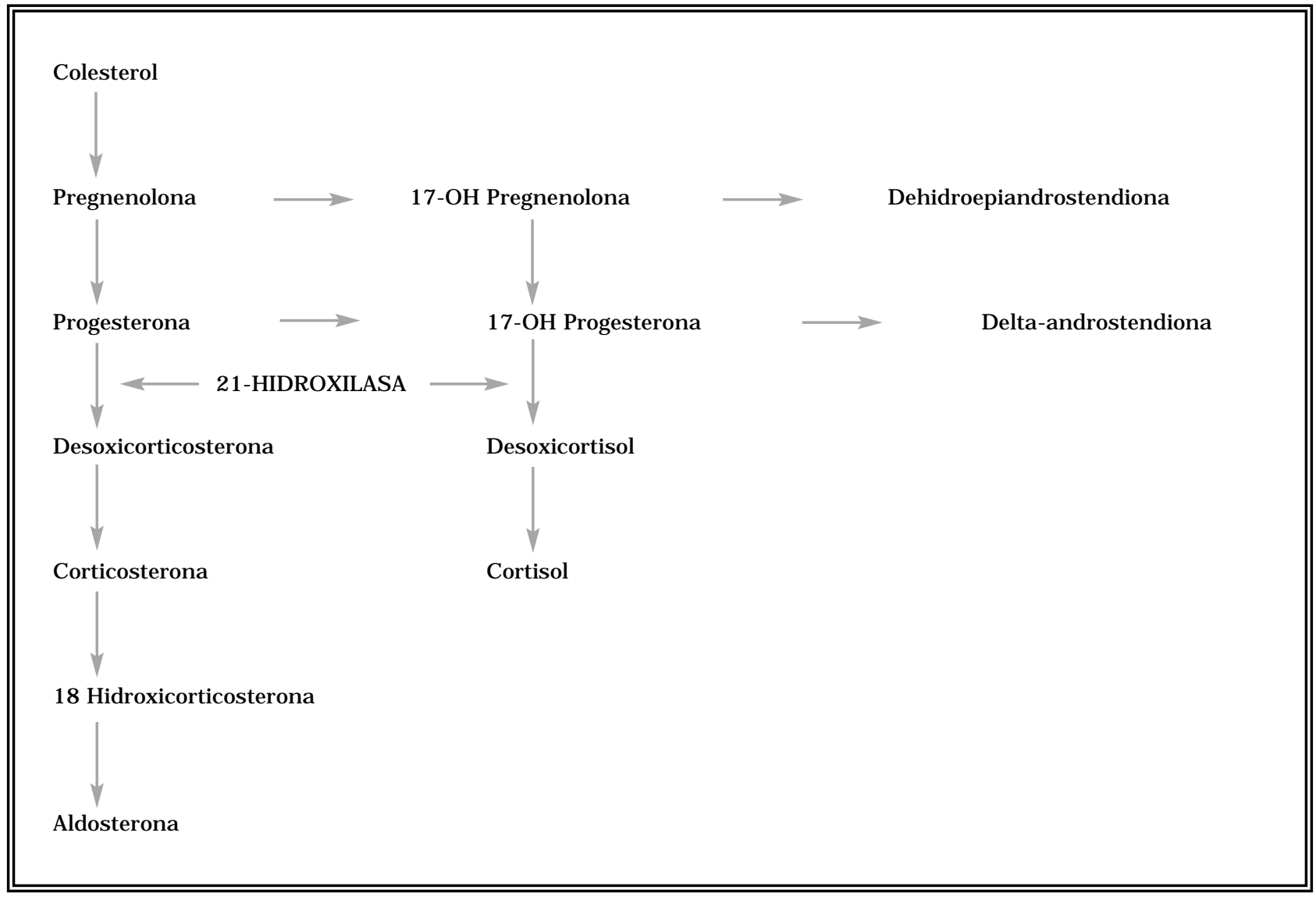

que sólo son diagnosticadas en estudios ecográficos, por lo que se deben realizar estos estudios periódicamente a estos pacientes ${ }^{3,5,7}$.

En otras ocasiones, como nuestro caso, se manifestó clínicamente como infertilidad junto a un aumento significativo del volumen testicular. La repercusión de esta hiperplasia de restos adrenales sobre la fertilidad es dificil de valorar ya que son pocos los casos descritos en la literatura ${ }^{4,5,8}$, y menos aún los que tienen una evolución muy larga sin tratamiento supresor adecuado. No obstante, en el estudio histológico de nuestro caso se observó una sustitución total del parénquima testicular por la hiperplasia de restos adrenales y sólo se observaron ocasionales túbulos seminíferos con paredes engrosadas, que mostraban marcada esclerosis e hialinización de su pared y sin apreciarse en ningún momento restos de células germinales en su interior, lo que justifica la infertilidad de este paciente.
Por otra parte, algunos autores han propugnado que el aumento de la 17-OH-progesterona en vena espermática junto a la disminución del tamano testicular cuando se instaura tratamiento supresor con corticoides es prácticamente diagnóstico de la hiperplasia de restos adrenales en el testículo ${ }^{4,5}$.

Histológicamente, sin embargo, es prácticamente imposible diferenciarlo del tumor de células de Leydig, sin la información clínica completa. Además en la literatura nacional e internacional no están bien definidos unos criterios histológicos que permitan diferenciar estas dos enfermedades con clínica, tratamiento y evolución tan distintos.

Sin embargo, la presencia de cristales de Reinke y la existencia muy focal de lipofucsina, parecen ser más frecuentes en el tumor de células de Leydig que en la hiperplasia de restos adrenales. De hecho, la gran dificultad para diagnosticar histológicamente la hiperplasia de restos adrenales en el testículo está ampliamente demos- 
trada en la literatura, ya que la mayor parte de ellos fueron etiquetados como tumor de células de Leydig $^{8}$ y en gran parte de ellos se realizó orquiectomía, incluso bilateral ${ }^{9}$.

Recientemente se han descrito algunos criterios histológicos que podrían contribuir al diagnóstico diferencial de estas dos lesiones: hiperplasia de restos adrenales versus tumor de células de Leydig ${ }^{10}$. Bock y cols. han demostrado que existen diferencias estadísticamente significativas entre algunos criterios histológicos al comparar ambas enfermedades, y señalan que el pleomorfismo nuclear, bajo índice mitótico, extensa fibrosis, acúmulos linfoides, abundante pigmento de lipofucsina, metaplasia de tejido adiposo y positividad para la sinaptofisina son más frecuentes en la hiperplasia de restos adrenales en el testículo que en el tumor de células de Leydig ${ }^{10}$.
En nuestro caso se observaron todos los criterios histológicos anteriormente comentados, excepto la metaplasia adiposa. Sin embargo, pensamos que todos estos datos deben ser confirmados en estudios posteriores y series más amplias.

En la Tabla III, describimos algunas características clínicas e histológicas que pueden contribuir a establecer el diagnóstico diferencial entre el tumor de células de Leydig y la hiperplasia de restos adrenales en el testículo.

Recientemente se ha descrito el primer caso de hiperplasia de restos adrenales en el ovario de una mujer de 36 años con hiperplasia suprarrenal congénita por déficit de 21-hidroxilasa. Clínicamente se manifestó por virilización y aumento de los niveles de testosterona, e histológicamente mostraba características histológicas idénticas a las de nuestro caso ${ }^{11}$.

\section{TABLA III}

CRITERIOS DIAGNÓSTICOS PARA DIFERENCIAR LA HIPERPLASIA DE RESTOS ADRENALES DEL TUMOR DE CÉLULAS DE LEYDIG

\begin{tabular}{|c|c|c|}
\hline Criterios clínicos & Hiperplasia de restos adrenales & Tumor de células de Leydig \\
\hline Localización & Bilateral y multifocal & Unilateral y unifocal \\
\hline Tamaño & Grande & Más pequeño \\
\hline Antecedentes clínicos & $\begin{array}{l}\text { Síndromes con aumento de } \\
\text { ACTH: } \\
\text { - Déficit de 21-hidroxilasa } \\
\text { - Enfermedad de Addison } \\
\text { - Síndrome de Cushing, etc. }\end{array}$ & Sin interés \\
\hline Clínica & $\begin{array}{l}\text { Asintomático } \\
\text { Infertilidad }\end{array}$ & $\begin{array}{l}\text { Ginecomastia } \\
\text { Disminución de la líbido }\end{array}$ \\
\hline \multirow[t]{2}{*}{ Otros datos } & $\begin{array}{l}\text { Disminución del tamaño testicular } \\
\text { con corticoides }\end{array}$ & \\
\hline & $\begin{array}{l}\text { Aumento de } 17 \mathrm{OH} \text { progesterona } \\
\text { en vena espermática }\end{array}$ & \\
\hline \multicolumn{3}{|l|}{ Criterios histológicos } \\
\hline Pleomorfismo nuclear & +++ & $+/++$ \\
\hline Índice mitótico & +++ & + \\
\hline Fibrosis & +++ & + \\
\hline Infiltrado linfoide & +++ & $-/+$ \\
\hline Metaplasia adiposa & +++ & - \\
\hline Lipofucsina & +++ & + \\
\hline Sinaptofisina & + & - \\
\hline
\end{tabular}


En resumen, pensamos que, a pesar de la gran dificultad diagnóstica para el patólogo de la hiperplasia de restos adrenales, pensamos que el diagnóstico definitivo de esta enfermedad se debe de realizar con una adecuada correlación clínica, radiológica e histológica, ya que no podemos descartar patología testicular neoplasia en pacientes con síndrome adrenogenital, como ha sido descrito ocasionalmente en estos pacientes ${ }^{12}$.

\section{REFERENCIAS}

1. WHITE PC, SPEISE PW.: Congenita adrenal hyperplasia due to 21-hydeoxylase deficiency. Endocr Rev 2000; 21 (3): 245-291.

2. OLIVER A, EZQUIETA B, JARIEGO CM, VARELA JM, GANCEDO PG, GRACIA R.: Analysis of the steroid 21hydroxylase gene in 65 children with hyperandrogenism. Horm Res 1997; 48 (suppl 2): 102.

3. ABO K, SUMINO K, NISHIO H, HOZUMI T, ISHIDA Y, FUIJIEDA K, TAJI KAZUMI T.: 21-hydroxylase deficiency presenting as massive bilateral a masses in the seventh decade of life. Endocr $J$ 1998; 46 (6): 817-823.

4. MOSTAFID H, HAWROCKI J, FLETCHER MS, VAUGHAN NJ, MELCHER DH.: The testicular tumour of adrenogenital syndrome: an unusual of male infertility. Br J Urol 1998; 81: 649-650.

5. CUTFIELD RG, BATEMAN JM, ODELL WD.: Infertility caused by bilateral testicular masses secondary to congenital adrenal hyperplasia (21-hydroxilase deficiency). Fertil Steril 1983; 40: 809-814.

6. CLARK RV, ALBERTSON BD, MUNABI A, CASSORLA F, AGUILERA G, WARREN DW et al.: Steroidogenic enzyme activities, morphology, and receptor studies of a testicular adrenal rest in a patient with congenital adrenal hyperplasia. J Clin Endocrinol Metab 1990; 70: 1408-1413.
7. SHAWKER TH, DOPPMAN JL, CHOYKE PL et al.: Intratesticular masses associated with abnormally functioning adrenal glands. J Clin Ultrasound 1992; 20: $51-58$.

8. WILSON BE, NETZLOFF ML.: Prymary testicular abnormalities causong precocious pubert cell tuor, Leydig cell hyperplasia, and adrenal rest tumor. Ann Clin Lab Sci 1983; 13 (4): 315-320.

9. CUNNAH D, PERRY L, DACIE JA, GRRANT DB, LOWE DG, SAVAGE MO, BESSER GM.: Bilateral testicular tumours in congenital adrenal hyperplasia: a continuing diagnostic and therapeutic dilemma. Clin Endocrinol 1989; 30 (2): 141-147.

10. BOCK BJ, CHEVILLE JC.: Diagnostic histologic features of testicular tumor of adrenogenital syndrome: differentiation from Leydig cell tumor. Unites States and Canadian Academy of Pathology International Academy. Annual meeting. New Orleans, Louisiana. Marzo 2000: 25-31.

11. HIKMAT AA, STANEK J, LIU J, MANGU PN, NIEMANN T, YOUNG RH.: Ovarian tumor of the adrenogenital syndrome. The first reported case. Am J Surg Pathol 2001; 25 (11): 1443-1450.

12. DAVIS JM, WOODROOF J, SADASIVAN R, STEPHENS R.: Case report: congenital adrenal hyperplasia and malignant Leydig cell tumor. Am J Med Sci 1995; 309: 63-65.

Dra. P. San Miguel Fraile

Servicio de Anatomía Patológica

Centro Médico POVISA

C/ Salamanca, 5

362211 Vigo (Pontevedra)

(Trabajo recibido el 1 abril de 2002) 